\title{
Aplicação da análise de componentes principais (ACP) no reconhecimento de agrupamentos químicos como ferramenta de pré-identificação de fases mineralógicas em amostras ambientais
}

\author{
Galvão, E. S. ${ }^{1 *}$; Orlando, M. T. D. ${ }^{1}$; Feroni, R. C. ${ }^{2}$; Passamai, J. L. Jr ${ }^{1}$ \\ 1 Departamento de Física, Universidade Federal do Espírito Santo, Vitória, ES, Brasil. \\ 2 Departamento de Engenharias e Tecnologia, Universidade Federal do Espírito Santo, São Mateus, ES, Brasil. \\ *e-mail: elsongalvao@gmail.com
}

\begin{abstract}
Resumo
A identificação de fases mineralógicas em amostras ambientais, tais como sedimentos, solo e material particulado atmosférico, se mostra um grande desafio à pesquisadores, especialmente aos menos experientes ou aqueles que não conheçam a composição mineralógica da matriz, devido tais amostras serem matrizes multifásicas. Nem sempre os softwares de reconhecimento de difratogramas são acessíveis à comunidade acadêmica, devido à falta de recursos para a obtenção de licenças. Como alternativa, é usual a identificação manual das fases através de fichas cristalográficas disponíveis em alguns repositórios. Entretanto, esse pode ser um processo subjetivo e demorado. Este trabalho propõe o uso de uma ferramenta estatística multivariada, a Análise de Componentes Principais (ACP), para o reconhecimento de padrões e agrupamentos químicos que possam tornar a identificação das fases minerais em amostras multifásicas mais objetiva. Os resultados da ACP para uma amostra ambiental de areia de praia mostram 6 agrupamentos distintos de elementos químicos que facilitam a inferência das fases mineralógicas mais prováveis presentes na matriz, sendo esses agrupamentos formados provavelmente por caulinita e muscovita, ilmenita, zirconita, monazita, branerita, e isoladamente o urânio.
\end{abstract}

\begin{abstract}
The identification of mineralogical phases in environmental samples, such as sediments, soil and atmospheric particulate matter, is a significant challenge for researchers, especially those who are less experienced or who do not know the mineralogical composition of the matrix, because of the multiphase nature. Diffractogram recognition software is not always accessible to the academic community because of the lack of resources to get licenses. As an alternative way, it is usually the manual identification through crystallographic files, available in some repositories. However, it may be a subjective and time-consuming process. This work proposes the use of Principal Component Analysis (PCA), as a statistical tool for recognizing patterns and chemical groupings, turning the identification of mineral phases in the samples more objective. The PCA results for an environmental sample of beach sand showed 6 distinct elemental groupings that made easier the inference of the most likely mineralogical phases in the matrix, probably composed of kaolinite and muscovite, ilmenite, zirconite, monazite, brannerite, and apart, uranium.
\end{abstract}

Keywords (Palavras chaves): Análise por componentes principais (ACP), fases mineralógicas, difração de raios-X.

\section{Introdução}

A difração de raios-X (DRX) tem sido amplamente utilizada na determinação de fases minerais presentes em amostras ambientais, tais como solo, sedimentos e material particulado atmosférico, com o objetivo de identificar possíveis fontes de contaminação ambiental. 
Orlando et al. (2020) [1] estudaram a composição mineralógica de sedimentos do Rio Doce por meio da difração ressonante de raios- $X$ por luz sincrotron, e identificaram grupos mineralógicos com base em ferro, compostos principalmente por hematita e magnetita, na qual estavam associados em grande parte aos rejeitos do rompimento da barragem de Fundão em Minas Gerais. Galvão et al. (2020) [2], também com uso da técnica de difração, identificaram fases mineralógicas incomuns no material particulado atmosférico na cidade de Vitória - ES, na qual tais fases incomuns forneceram informações importantes sobre as prováveis fontes de emissão dessas fases minerais na atmosfera. Em ambos os casos apresentados, a identificação de fases mineralógicas nas amostras permitiu a identificação das atividades associadas à poluição ambiental, o que permite a identificação dos responsáveis e a proposição de políticas públicas de controle e mitigação da poluição ambiental.

De acordo com Ladd e Palmer (2013)[3], a determinação de fases cristalinas na difração de amostras em pó pode gerar erros de interpretação devido a sobreposição de picos da reflexão de raios- $X$. Especialmente em amostras ambientais, nas quais dezenas de fases fazem parte da composição mineralógica do material, tais sobreposições podem conduzir a erros de interpretação como também superestimar o teor de fases com picos sobrepostos, conforme reportado por Patey et al.(2015) [4].

De acordo com Gotelli e Ellison (2011) [5], técnicas estatísticas multivariadas como a ACP permitem o agrupamento de amostras e a identificação de grupos químicos similares em classes, permitindo que essas sejam identificadas e interpretadas separadamente. É esperado correlações entre algumas espécies presentes nos dados originais; por exemplo, o ferro com o oxigênio em amostras contendo hematita (minérios de ferro), ou o silício com o alumínio em amostras contendo feldspatos (solos). Nesses casos, a ACP pode auxiliar na interpretação dos difratogramas, uma vez que, a presença ou ausência de determinados elementos pode servir como filtro de possíveis fases presentes nas amostras. A ACP reduz um grande número de variáveis originais correlacionadas para um número pequeno de variáveis novas e não correlacionadas [5]. Portanto, em amostras ambientais, nas quais o número de elementos químicos e fases minerais pode ser elevado, a ACP pode facilitar a interpretação dos dados.

\section{Metodologia}

Amostras de areia da praia de Meaípe, localizada no estado do Espírito Santo, foram analisadas por meio de fluorescência de raios-X para determinação da composição elementar. As amostras foram analisadas por meio de um espectrômetro EDXRF modelo 720 (Shimadzu, Japão).

O programa Origin 9.0 (OriginLab) foi utilizado para a análise do conjunto de dados, incluindo a estatística descritiva e a análise multivariada por ACP.

\section{Discussão dos resultados}

A Tabela 1 mostra a composição elementar da areia de Meaípe. Os elementos majoritários são o silício (Si) e zircônio ( $Z r$ r), com concentrações iguais a $37,2 \%$ e 19,9\%, respectivamente. Em seguida estão o titânio (Ti $=4,53 \%)$, alumínio $(\mathrm{Al}=3,63 \%)$, Neodímio $(\mathrm{Nd}=$ $3,89 \%$ ) e cério ( $\mathrm{Ce}=3,56 \%$ ), além de ferro $(\mathrm{Fe})$ e tório (Th), ambos com 1,33\%. Dentre os elementos com concentração traço, estão o háfnio $(\mathrm{Hf}=0,62 \%)$ e o urânio $(U=0,03 \%)$.

\begin{tabular}{ccccc}
\multicolumn{5}{c}{ Tabela 1. Composição elementar (\%) da areia de Meaípe. } \\
\hline Elemento & Média & Mínimo & Máximo & $\begin{array}{c}\text { Desvio } \\
\text { Padrão }\end{array}$ \\
\hline $\mathrm{Si}$ & 37,2 & 9,67 & 81,1 & 37,2 \\
$\mathrm{Zr}$ & 19,9 & 1,63 & 32,2 & 8,10 \\
$\mathrm{Ti}$ & 4,53 & 0,03 & 10,4 & 4,53 \\
$\mathrm{Al}$ & 3,63 & 0,01 & 14,5 & 3,64 \\
$\mathrm{Nd}$ & 3,89 & 0,01 & 17,6 & 5,04 \\
$\mathrm{Ce}$ & 3,56 & 0,01 & 21,2 & 5,80 \\
$\mathrm{Th}$ & 1,33 & 0,11 & 3,07 & 0,85 \\
$\mathrm{Fe}$ & 1,32 & 0,01 & 2,88 & 1,32 \\
$\mathrm{Hf}$ & 0,62 & 0,01 & 1,03 & 0,28 \\
$\mathrm{U}$ & 0,03 & 0,01 & 0,06 & 0,03 \\
\hline
\end{tabular}

Diversas são as combinações possíveis entre os elementos acima, dificultando a inferência das possíveis fases cristalográficas presentes na amostra de areia. A análise de correlação linear de Pearson se mostrou adequada apenas para a relação entre Th e $\mathrm{Y}$, conforme Figura 1.

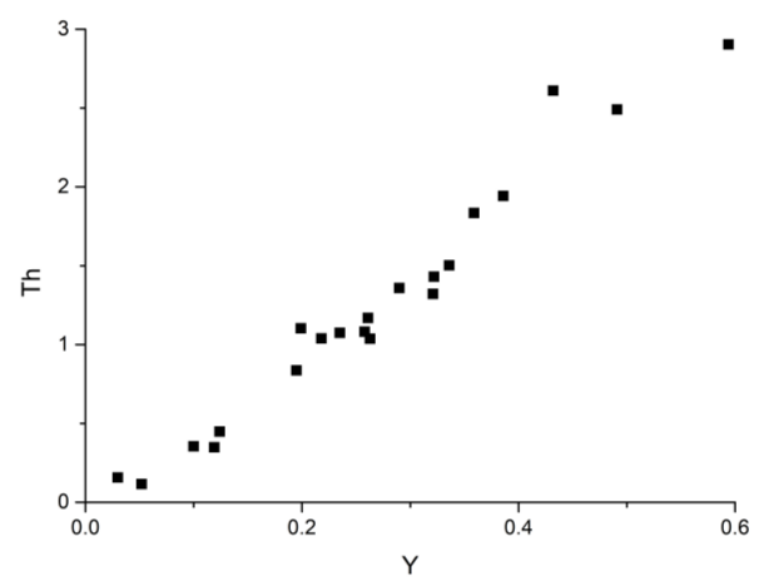

Figura 1. Correlação de Pearson entre o Tório e Ítrio. 
Entretanto, para os demais elementos, as matrizes de dispersão não mostram correlações que permitam inferir agrupamentos que representem com acurácia as prováveis fases minerais presente nas amostras. Isso se dá pelo fato de que esse tipo de análise estatística não mostra as correlações entre todas as 10 espécies presentes nas amostras de areia, mas apenas as correlações lineares entre duas espécies, uma característica incompatível com amostras ambientais.

A Figura 2 mostra a análise de ACP para as 10 espécies químicas presentes nas amostras analisadas por EDXRF. Conforme mostrado na Figura 2, existem 6 grupos distintos formados pelo agrupamento de diferentes elementos. Na ACP, quanto menor o ângulo formado pelas distâncias euclidianas entre as variáveis, maior é o grau de correlação entre essas espécies e, portanto, pode indicar quais elementos estão mais fortemente correlacionados com algum mineral específico. A análise por ACP na Figura 2 explica aproximadamente $67 \%$ da variância dos dados, mostrando agrupamentos formados por elementos mais fortemente correlacionados.

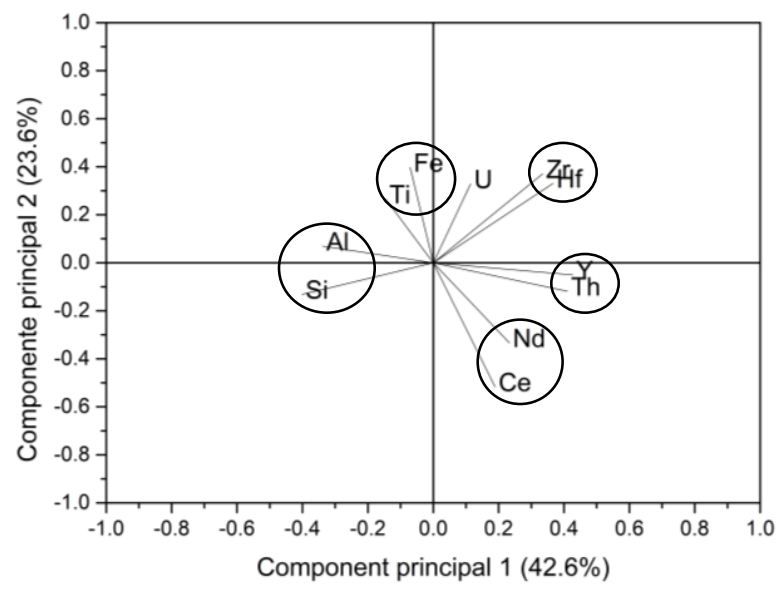

Figura 2. Análise de componentes principais dos dados de composição química das areias de Meaípe.

A Figura 2 revelou 6 agrupamentos distintos formados por: (1) Al e Si, indicando a presença de quartzo (SiO2) e argilominerais como caulinita $\left(\mathrm{Al}_{2} \mathrm{Si}_{2} \mathrm{O}_{5}(\mathrm{OH})_{4}\right)$, além

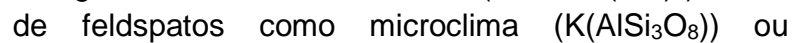
filosilicatos como muscovita $\left(\mathrm{KAl}_{2}\left(\mathrm{Si}_{3} \mathrm{Al}\right) \mathrm{O}_{10}(\mathrm{OH})_{2}\right)$; (2) $\mathrm{Ti}$ e $\mathrm{Fe}$, indicando a presença de ilmenita $\left(\mathrm{FeTiO}_{3}\right)$; (3) $\mathrm{Zr}$ e $\mathrm{Hf}$, provavelmente associado à zirconita $\left(\mathrm{ZrSiO}_{4}\right)$, - que explica a variação contrária entre esses elementos e o silício, na qual maiores concentrações de $\mathrm{Zr}\left(\mathrm{ZrO}_{2}\right)$ e $\mathrm{Hf}\left(\mathrm{HfO}_{2}\right)$ no cristal tendem a diminuir a contribuição de sílica $\left(\mathrm{SiO}_{2}\right) ;$ (4) Y e Th, assim como $\mathrm{Nd}$ e $\mathrm{Ce}$, possivelmente associados à monazita ((Ce, $\mathrm{La}, \mathrm{Nd}, \mathrm{Th}, \mathrm{Y}) \mathrm{PO}_{4}$ ); e (5) $\mathrm{U}$, aparecendo isolado dos demais elementos, mas apresentando certa proximidade com o grupo ( $\mathrm{Fe}, \mathrm{Ti})$, indicando a provável presença de branerita $\left((\mathrm{U})(\mathrm{Ti}, \mathrm{Fe})_{2} \mathrm{O}_{6}\right)$.

\section{Conclusão}

A análise por ACP dos dados gerados pela fluorescência forneceu importante informação a respeito dos agrupamentos formados pelos elementos mais fortemente correlacionados, indicando as prováveis fases mineralógicas presente numa amostra ambiental multifásica de areia de praia. Tal processo agilizou a busca de fichas cristalográficas específicas para o processo de análise dos difratogramas, tornando o processo menos subjetivo, mais ágil e acurado.

\section{Agradecimentos}

Os autores agradecem a CAPES pelo apoio financeiro.

\section{Referências}

[1] ORLANDO M. T. D. et al., "Tracing iron ore tailings in the marine environment: An investigation of the Fundão dam failure," Chemosphere, vol. 257, p. 127184, 2020, doi: https://doi.org/10.1016/j.chemosphere.2020.12718 4.

[2] GALVÃO E. S., ORLANDO M. T. D., SANTOS J. M., and LIMA A. T., "Uncommon chemical species in PM2.5 and PM10 and its potential use as industrial and vehicular markers for source apportionment studies," Chemosphere, p. 124953, Sep. 2020, doi: 10.1016/J.CHEMOSPHERE.2019.124953.

[3] LADD M. and PALMER R., Structure Determination by X-ray Crystallography, 5th ed. Springer US, 2013.

[4] PATEY M. D., ACHTERBERG E. P., RIJKENBERG M. J., and PEARCE R., "Aerosol time-series measurements over the tropical Northeast Atlantic Ocean: Dust sources, elemental composition and mineralogy," Mar. Chem., vol. 174, pp. 103-119, 2015, doi: https://doi.org/10.1016/j.marchem.2015.06.004.

[5] GOTELLI N. J. and ElLISON A. M., Princípios de estatística em ecologia. Porto Alegre: Artmed, 2011. 